\title{
Um Algoritmo Dual Viável para Programação Linear $^{1}$
}

M.A.F. MENEZES', Departamento de Computação, Universidade Católica de Goiás (UCG), Cx.P. 86, 74605-010 Goiânia, GO, Brasil.

Resumo Neste trabalho estudamos um algoritmo de ponto-interior-inviável dual viável para programação linear devido a Menezes e Gonzaga [3], desenvolvendo uma estratégia preditora-corretora sob uma certa condição associada à viabilidade primal, com o objetivo de melhorar a eficiência do algoritmo mantendo a sua complexidade em iterações.

\section{Introdução}

Existe um problema em aberto em Programação Linear (PL) que consiste na obtenção de um algoritmo de ponto-interior-inviável com complexidade polinomial em $O(\sqrt{n} L)$ iterações para a formulação não artificial do problema de PL, onde $n$ é o número de variáveis do problema e $L$ é o tamanho do problema para dados inteiros. Com a exceção do método homogêneo e auto-dual para PL (Ye, Todd e Mizuno [8]) que possui $O(\sqrt{n} L)$ iterações para uma formulação artificial do problema de PL (e para problemas de Complementaridade Linear com a hipótese de monotonicidade, LCP, Ye [7]), a menor complexidade em iterações para a formulação não artificial é $O(n L)$ (por exemplo, veja Sheng e Potra [5]).

Em [6] e em [3] estudou-se este problema com a hipótese de viabilidade dual, obtendo-se, em ambos, algoritmos com $O(\sqrt{n} L)$ iterações. Em [1] estudou-se métodos primais-duais com inviabilidade primal e viabilidade dual, mas não se tratou sobre complexidade.

Neste trabalho estudamos e melhoramos o algoritmo em [3] através de um algoritmo que permite passos preditor-corretor sob 'certa condição'. Na inicialização do nosso algoritmo é necessário um procedimento de centralização, que é feito em geral nos algoritmos de pontos interiores (viáveis). Este procedimento de centralização tem complexidade $O(\sqrt{n} L)$ iterações (veja Gonzaga [2]).

Definiremos o problema na próxima seção. Na seção 3, definiremos as medidas de proximidade e o passo de Newton e estabeleceremos uma ponte entre os problemas de viabilidade e otimalidade. O algoritmo, sua convergência e sua complexidade em iterações serão formalizados na última seção.

\footnotetext{
${ }^{1}$ Este trabalho teve o apoio da Pró-Reitoria de Pós-Graduação e Pesquisa da Universidade Católica de Goiás (PROPE/UCG).

2 marco@ucg.br
} 
$\mathrm{O}$ vetor $e$ denota o vetor de uns e 0 denota o vetor nulo, ambos, com dimensões apropriadas no contexto. Denotamos operações componente por componente pela notação usual de números reais. Assim, dados vetores $u, v$ com a mesma dimensão, $u v, u / v=u v^{-1}$, etc. denotam os vetores com componentes $u_{j} v_{j}, u_{j} / v_{j}$, etc.. Finalmente, os sub-índices ' 1 ' e '2' referem-se à viabilidade e à otimalidade, respectivamente.

\section{O Problema}

Consideremos os números inteiros $m$ e $n$ tais que $n>m>0$. Dados uma matriz $\tilde{A} \in R^{m \times(n-1)}$ e vetores $b \in R^{m}$ e $\tilde{c} \in R^{n-1}$, denominamos de problema original o seguinte problema de programação linear primal:

$$
\begin{array}{llrl}
(\tilde{P}) & \text { minimizar } & \tilde{c}^{T} \tilde{x} \\
& \text { sujeito a: } & \tilde{A} \tilde{x} & =b \\
& & \tilde{x} & \geq 0 .
\end{array}
$$

Sem perda de generalidade supomos que $\tilde{A}$ tem posto igual a $m$.

As hipóteses para este trabalho são:

(H1) O conjunto de soluções ótimas de $(\tilde{P})$ é não vazio e limitado.

(H2) Viabilidade dual.

Dado $\tilde{x}^{0} \geq 0$ definimos

$$
A=\left[\begin{array}{ll}
\tilde{A} & b-\tilde{A} \tilde{x}^{0}
\end{array}\right], \quad c^{T}=\left[\tilde{c}^{T}, 0\right] \text { e } \xi^{T}=\left[0^{T}, 1\right] .
$$

Observamos que o ponto $x^{T}=\left[\left(\tilde{x}^{0}\right)^{T}, 1\right]$ satisfaz $A x=b$ e $x \geq 0$. Além disso, a matriz $A$ preserva o mesmo posto de $\tilde{A}, \xi^{T} x=x_{n}$ e $c^{T} x=\tilde{c}^{T} \tilde{x}$.

Por construção, um problema equivalente ao problema $(\tilde{P})$, a menos de uma dimensão, é o seguinte:

$$
\begin{array}{cc}
\left(P_{0}\right) \quad \text { minimizar } & c^{T} x \\
\text { sujeito a: } & A x=b \\
& \xi^{T} x=0 \\
& x \geq 0 .
\end{array}
$$

O seu dual é

$$
\begin{array}{lcc}
\left(D_{0}\right) & \text { maximizar } & b^{T} y \\
& \text { sujeito a: } & A^{T} y-\xi t+s=c \\
& & s \geq 0 .
\end{array}
$$

Através da hipótese $(H 1)$ vamos introduzir um limitante inferior para o parâmetro $\theta_{c}$, a saber: dado $\theta_{0} \geq 1$,

$$
v\left(P_{2}\right)_{\theta_{0}}=\min \left\{c^{T} x ; A x=b, \xi^{T} x \leq \theta_{0}, x \geq 0\right\} .
$$


Vamos resolver o par de problemas $\left(P_{0}\right)$ e $\left(D_{0}\right)$ considerando dois outros problemas (subproblemas): dados $\theta_{c}>v\left(P_{2}\right)_{\theta_{0}}$ e $\theta>0$, definimos

$$
\begin{array}{ll}
\left(P_{1}\right) \quad \text { minimizar } & \xi^{T} \breve{x} \\
\text { sujeito a: } & A \breve{x}=b \\
& c^{T} \breve{x}+\breve{x}_{n+1}=\theta_{c} \\
& \breve{x} \geq 0, \breve{x}_{n+1} \geq 0
\end{array}
$$

e

$$
\begin{array}{ll}
\left(P_{2}\right) \quad \text { minimizar } & c^{T} x \\
\text { sujeito a: } & A x=b \\
& \xi^{T} x+x_{n+1}=\theta \\
& x \geq 0, x_{n+1} \geq 0,
\end{array}
$$

e os seus respectivos problemas duais

$$
\begin{array}{cc}
\left(D_{1}\right) \quad \text { maximizar } & b^{T} y-\theta_{c} t \\
\text { sujeito a: } & A^{T} y-c t+s=\xi \\
& t \geq 0, s \geq 0,
\end{array}
$$

$\mathrm{e}$

$$
\begin{array}{ccc}
\left(D_{2}\right) \quad \text { maximizar } & b^{T} \omega-\theta l \\
& \text { sujeito a: } & A^{T} \omega-\xi l+r=c \\
& l \geq 0, r \geq 0 .
\end{array}
$$

Para $\theta_{c}>v\left(P_{2}\right)_{\theta_{0}}$, denotamos

$$
\begin{gathered}
\mathcal{X}_{\theta_{c}}^{0}=\left\{\left(\breve{x}, \breve{x}_{n+1}\right) \in R_{++}^{n} \times R_{++} ; A \breve{x}=b, c^{T} \breve{x}+\breve{x}_{n+1}=\theta_{c}\right\}, \\
\hat{\mathcal{S}}_{\theta_{c}}^{0}=\left\{\left(\breve{s}, \breve{s}_{n+1}\right) \in R_{++}^{n} \times R_{++} ; A^{T} \breve{y}-c \breve{t}+\breve{s}=\xi, \breve{t}=\breve{s}_{n+1} \text {, para algum } \breve{y} \in R^{m}\right\}, \\
\hat{\mathcal{F}}_{\theta_{c}}^{0}=\left\{\left(\breve{x}, \breve{x}_{n+1}, \breve{s}, \breve{s}_{n+1}\right) \in \mathcal{X}_{\theta_{c}}^{0} \times \hat{\mathcal{S}}_{\theta_{c}}^{0}\right\} \\
\text { e, para } \theta>0, \operatorname{denotamos} \\
\mathcal{X}_{\theta}^{0}=\left\{\left(x, x_{n+1}\right) \in R_{++}^{n} \times R_{++} ; A x=b, \xi^{T} x+x_{n+1}=\theta\right\}, \\
\hat{\mathcal{S}}_{\theta}^{0}=\left\{\left(s, s_{n+1}\right) \in R_{++}^{n} \times R_{++} ; A^{T} y-\xi t+s=c, t=s_{n+1}, \text { para algum } y \in R^{m}\right\}, \\
\hat{\mathcal{F}}_{\theta}^{0}=\left\{\left(x, x_{n+1}, s, s_{n+1}\right) \in \mathcal{X}_{\theta}^{0} \times \hat{\mathcal{S}}_{\theta}^{0}\right\} .
\end{gathered}
$$

O par de problemas $\left(P_{1}\right)$ e $\left(D_{1}\right)$ é o problema primal-dual associado ao problema de viabilidade primal-dual, enquanto o par de problemas $\left(P_{2}\right)$ e $\left(D_{2}\right)$ é o problema primal-dual associado ao problema de otimalidade primal-dual. A seguir 
vamos buscar uma formulação que envolve a resolução de sistemas de igualdades e desigualdades para estes pares de problemas.

Considere $\theta_{c}>v\left(P_{2}\right)_{\theta_{0}}$ e $\mu_{\xi}>0$. As condições de otimalidade para o problema que define o ponto central $\left(\breve{x}, \breve{x}_{n+1}\right) \in \mathcal{X}_{\theta_{c}}^{0}$ são as seguintes: existem $\bar{y} \in R^{m}$ e $\bar{t} \in R$ tais que definindo

$$
\breve{s}=\mu_{\xi} \breve{x}^{-1}>0 \text { e } \breve{s}_{n+1}=\frac{n \mu_{\xi}}{\breve{x}_{n+1}}>0
$$

$\breve{y}=\mu_{\xi} \bar{y}$ e $\breve{t}=-\mu_{\xi} \bar{t}$, obtemos o sistema primal-dual perturbado

$$
\begin{array}{rlrl}
A \breve{x} & & =b \\
c^{T} \breve{x}+\breve{x}_{n+1} & =\theta_{c} \\
A^{T} \breve{y}-c \breve{t}+\breve{s} & = \\
(P D)_{1} \quad \breve{s}_{n+1} & =0 \\
\breve{x} \breve{s} & =\mu_{\xi} e \\
\breve{x}_{n+1} \breve{s}_{n+1} & =n \mu_{\xi} \\
\breve{x}, \breve{s} \geq 0, \breve{x}_{n+1}, \breve{s}_{n+1} \geq 0 . &
\end{array}
$$

Observamos que o gap de dualidade associado a $\left(\breve{x}, \breve{x}_{n+1}\right)$ e $\left(\breve{y}, \breve{t}, \breve{s}, \breve{s}_{n+1}\right)$, satisfazendo o sistema primal-dual perturbado $(P D)_{1}$, é definido por

$$
\Delta_{1}=\xi^{T} \breve{x}-\left(b^{T} \breve{y}-\theta_{c} \breve{t}\right)=\breve{x}^{T} \breve{s}+\breve{x}_{n+1} \breve{s}_{n+1}=2 n \mu_{\xi} .
$$

Considere $\theta>0$ e $\mu>0$. As condições de otimalidade para o problema que define o ponto central $\left(x, x_{n+1}\right) \in \mathcal{X}_{\theta}^{0}$ são as seguintes: existem $\tilde{y} \in R^{m}$ e $\tilde{t} \in R$ tais que definindo

$$
s=\mu x^{-1}>0 \text { e } s_{n+1}=\frac{n \mu}{x_{n+1}}>0
$$

$y=\mu \tilde{y}$ e $t=-\mu \tilde{t}$, obtemos o sistema primal-dual perturbado

$$
\begin{array}{rlrl}
A x & & = \\
\xi^{T} x+x_{n+1} & & \theta \\
A^{T} y-\xi t+s & = & c \\
(P D)_{2}-t+s_{n+1} & = & 0 \\
x s \quad & = & \mu e \\
x_{n+1} s_{n+1} & = & n \mu \\
x, s \geq 0, x_{n+1}, s_{n+1} \geq 0 . &
\end{array}
$$

Observamos que o gap de dualidade associado a $\left(x, x_{n+1}\right)$ e $\left(y, t, s, s_{n+1}\right)$, satisfazendo o sistema primal-dual perturbado $(P D)_{2}$, é definido por

$$
\Delta_{2}=c^{T} x-\left(b^{T} y-\theta t\right)=x^{T} s+x_{n+1} s_{n+1}=2 n \mu .
$$




\section{As Medidas de Proximidade e o Passo de New- ton}

Dados $\theta_{c}>v\left(P_{2}\right)_{\theta_{0}}$ e $\mu_{\xi}>0$ e considerando o sistema $(P D)_{1}$, gostaríamos de encontrar $\left(\breve{x}, \breve{x}_{n+1}, \breve{s}, \breve{s}_{n+1}\right)$ tal que $\breve{x} \breve{s} / \mu_{\xi}=e$ e $\breve{x}_{n+1} \breve{s}_{n+1} / \mu_{\xi}=n$. Assim, definimos a medida de proximidade,

$$
\left(\breve{x}, \breve{x}_{n+1}, \breve{s}, \breve{s}_{n+1}, \mu_{\xi}\right) \in \hat{\mathcal{F}}_{\theta_{c}}^{0} \times R_{++} \mapsto \delta_{\theta_{c}}\left(\breve{x}, \breve{x}_{n+1}, \breve{s}, \breve{s}_{n+1}, \mu_{\xi}\right)=\delta_{\theta_{c}}
$$

onde

$$
\delta_{\theta_{c}}=\left\|\frac{1}{\mu_{\xi}}\left(\begin{array}{c}
\breve{x} \breve{s} \\
\frac{\breve{x}_{n+1} \breve{s}_{n+1}}{\sqrt{n}}
\end{array}\right)-\left(\begin{array}{c}
e \\
\sqrt{n}
\end{array}\right)\right\| .
$$

Da mesma forma, dados $\theta>0$ e $\mu>0$ e considerando o sistema $(P D)_{2}$, gostaríamos de encontrar $\left(x, x_{n+1}, s, s_{n+1}\right)$ tal que $x s / \mu=e$ e $x_{n+1} s_{n+1} / \mu=n$. Assim, definimos a medida de proximidade,

$$
\left(x, x_{n+1}, s, s_{n+1}, \mu\right) \in \hat{\mathcal{F}}_{\theta}^{0} \times R_{++} \mapsto \delta_{\theta}\left(x, x_{n+1}, s, s_{n+1}, \mu\right)=\delta_{\theta},
$$

onde

$$
\delta_{\theta}=\left\|\frac{1}{\mu}\left(\begin{array}{c}
x s \\
\frac{x_{n+1} s_{n+1}}{\sqrt{n}}
\end{array}\right)-\left(\begin{array}{c}
e \\
\sqrt{n}
\end{array}\right)\right\| .
$$

Consideremos $\alpha \in(0,1)$. Dados $\theta_{c}>v\left(P_{2}\right)_{\theta_{0}}$ e $\mu_{\xi}>0$, dizemos que o ponto $\left(\breve{x}, \breve{x}_{n+1}, \breve{s}, \breve{s}_{n+1}\right) \in \hat{\mathcal{F}}_{\theta_{c}}^{0}$ é aproximadamente central ou $\alpha$-central se, e somente se, $\delta_{\theta_{c}} \leq \alpha$. Por outro lado, dados $\theta>0$ e $\mu>0$, o ponto $\left(x, x_{n+1}, s, s_{n+1}\right) \in \hat{\mathcal{F}}_{\theta}^{0}$ é aproximadamente central ou $\alpha$-central se, e somente se, $\delta_{\theta} \leq \alpha$.

Agora vamos estabelecer a ponte entre os pares de problemas $\left(P_{1}\right),\left(D_{1}\right)$ e $\left(P_{2}\right)$, $\left(D_{2}\right)$ para pontos aproximadamente centrais.

Lema 3.1. Considere $\alpha=0,25$. Seja $\left(x, x_{n+1}, s, s_{n+1}\right) \in \hat{\mathcal{F}}_{\theta}^{0}$ um ponto aproximadamente central e sejam dados $\theta>0$ e $\mu>0$. Então, o ponto $\left(\breve{x}, \breve{x}_{n+1}, \breve{s}, \breve{s}_{n+1}\right)$ em $\hat{\mathcal{F}}_{\theta_{c}}^{0}$ é aproximadamente central para

$$
\begin{gathered}
\theta_{c}=c^{T} x+n \mu, \mu_{\xi}=\frac{x_{n+1}}{n}, \\
\breve{x}=x, \breve{x}_{n+1}=n \mu, \\
\breve{s}=\frac{s}{s_{n+1}} \text { e } \breve{s}_{n+1}=\frac{1}{s_{n+1}} .
\end{gathered}
$$

Demonstração. Lema 5.3.2 em [3]. 
Métodos de ponto-interior-inviável trabalham com pontos próximos da superfície de centros, conforme Mizuno, Todd e Ye [4]. Consideremos o problema $(P D)_{2}$. Dados os parâmetros $\alpha \in(0,1)$ (usualmente $\alpha=0,25)$ e $\theta>0$, a vizinhança da superfície de centros é definida por

$$
\mathcal{V}(\alpha, \theta)=\left\{\left(x, x_{n+1}, s, s_{n+1}, \mu\right) \in \hat{\mathcal{F}}_{\theta}^{0} \times R_{++} ; \delta_{\theta}\left(x, x_{n+1}, s, s_{n+1}, \mu\right) \leq \alpha\right\}
$$

Consideremos o problema $(P D)_{2}$. Dados $\theta>0,\left(x, x_{n+1}, s, s_{n+1}\right) \in \hat{\mathcal{F}}_{\theta}^{0}$ e $\mu>0$, consideremos os parâmetros $\nu \in R$ e $\gamma \geq 0$ associados aos parâmetros $\theta$ e $\mu$, respectivamente. Idealmente, gostaríamos de encontrar

$$
\begin{gathered}
x^{+}=x+u, \quad x_{n+1}^{+}=x_{n+1}+u_{n+1}, \\
y^{+}=y+w, \quad t^{+}=t+\Delta t, \quad s^{+}=s+v \text { e } s_{n+1}^{+}=s_{n+1}+v_{n+1},
\end{gathered}
$$

tais que $\left(x^{+}, x_{n+1}^{+}, s^{+}, s_{n+1}^{+}\right) \in \hat{\mathcal{F}}_{\nu \theta}^{0}, x^{+} s^{+}=\gamma \mu e$ e $x_{n+1}^{+} s_{n+1}^{+}=n \gamma \mu$.

O passo de Newton resolve isto aproximadamente linearizando o sistema primaldual perturbado $(P D)_{2}$, da seguinte maneira:

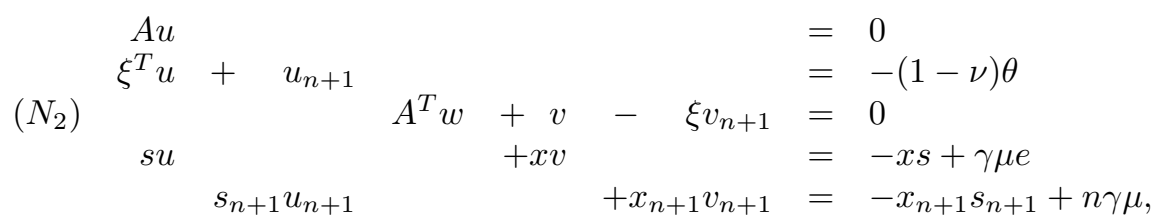

onde $\Delta t=v_{n+1}$.

Essencialmente, dizemos que a importância da disciplina pontos interiores é a garantia de eficiência do passo de Newton para alguma medida de proximidade. Neste sentido, segue-se o próximo resultado.

Lema 3.2. Considere $\sigma=0,1 / \sqrt{n}$. Dados o ponto $\left(x, x_{n+1}, s, s_{n+1}\right) \in \hat{\mathcal{F}}_{\theta}^{0}, \theta>0$ e $\mu>0$ tais que $\delta_{\theta}\left(x, x_{n+1}, s, s_{n+1}, \mu\right)=\delta_{\theta}<1$ e seja o resultado do passo de Newton dado por $\left(x^{+}, x_{n+1}^{+}, s^{+}, s_{n+1}^{+}\right)$a partir do ponto $\left(x, x_{n+1}, s, s_{n+1}\right)$. Então,

$$
\delta_{\theta}\left(x^{+}, x_{n+1}^{+}, s^{+}, s_{n+1}^{+}, \mu\right) \leq \frac{\delta_{\theta}^{2}}{\sqrt{8}\left(1-\delta_{\theta}\right)}
$$

$e\left(x^{+}, x_{n+1}^{+}, s^{+}, s_{n+1}^{+}\right) \in \hat{\mathcal{F}}_{\theta}^{0}$ para $\delta_{\theta} \leq 0,7$.

Demonstração. Para $\nu=1$, este é o caso do Teorema 5.4.1 em [3]. Caso contrário, este é o Teorema 2.4 em Sheng e Potra [5].

Agora vamos verificar o teste que é crucial para os dois algoritmos que apresentaremos na próxima seção, isto é, 'se'

$$
\breve{x}_{n}-\Delta_{1}=b^{T} \breve{y}-\theta_{c} \breve{t} \leq 0
$$


Usando o lema anterior, para pontos aproximadamente centrais, $\breve{y}=\frac{y}{s_{n+1}} \mathrm{e}$, também, $\breve{t}=\frac{1}{s_{n+1}}$. Daí,

$$
b^{T} y \leq \theta_{c}
$$

é o nosso teste considerando pontos aproximadamente centrais.

\section{O Algoritmo}

Inicialmente, vamos enunciar um algoritmo de ponto-interior-inviável dual viável de passos curtos, com $O(\sqrt{n} L)$ iterações e que necessita de um procedimento de centralização na sua inicialização, conforme [3].

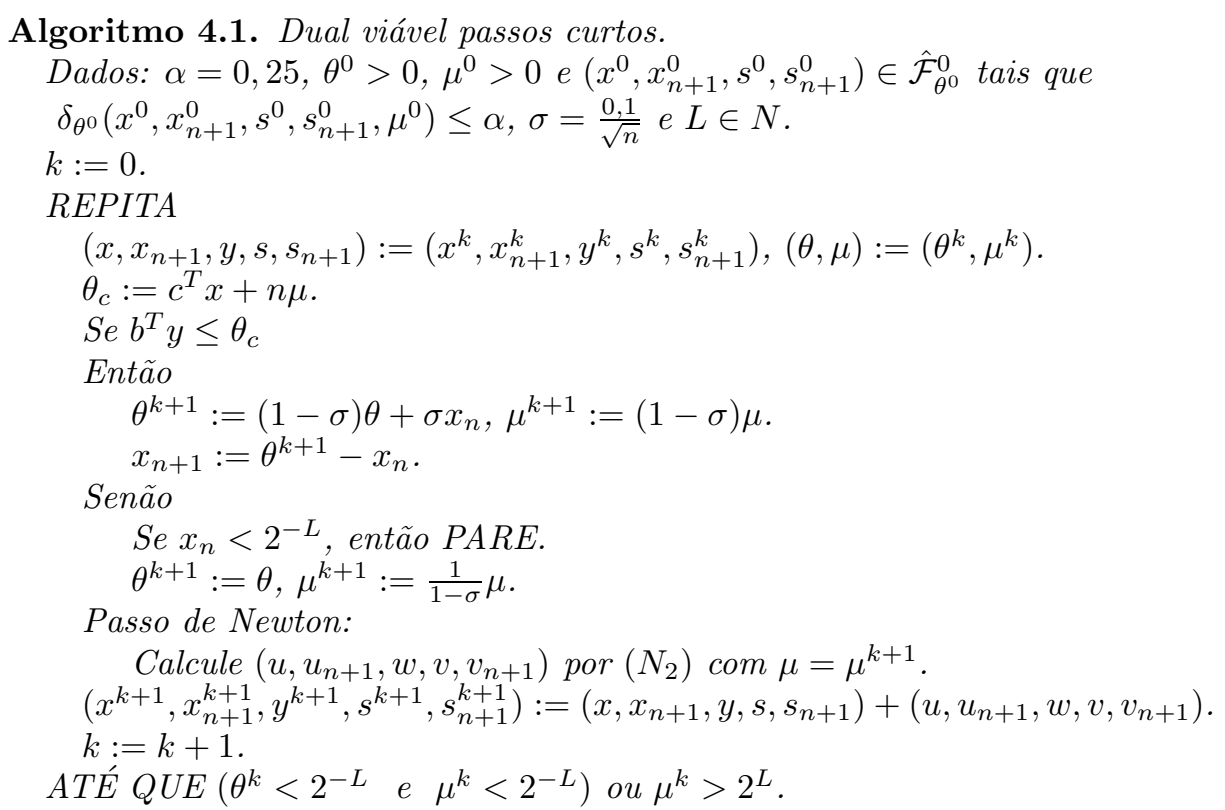

Finalizamos esta seção enunciando e demonstrando os resultados acerca do próximo algoritmo dual viável de passos não tão curtos, o qual mantém a mesma complexidade em iterações e que, também, necessita de um procedimento de centralização na sua inicialização.

Algoritmo 4.2. Dual viável.

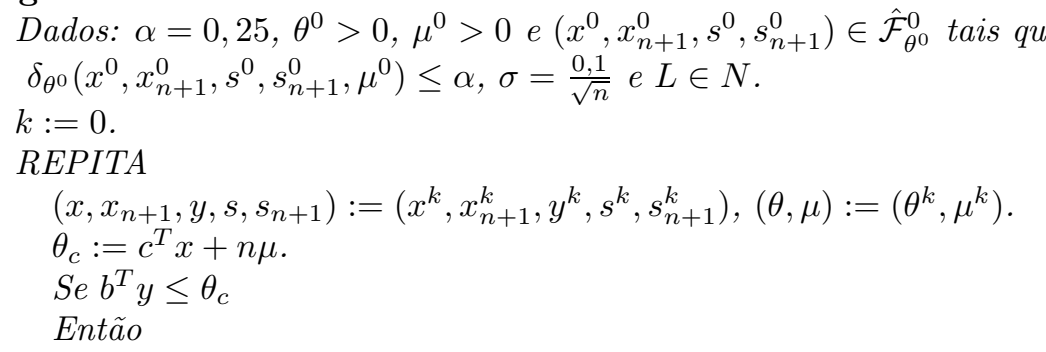




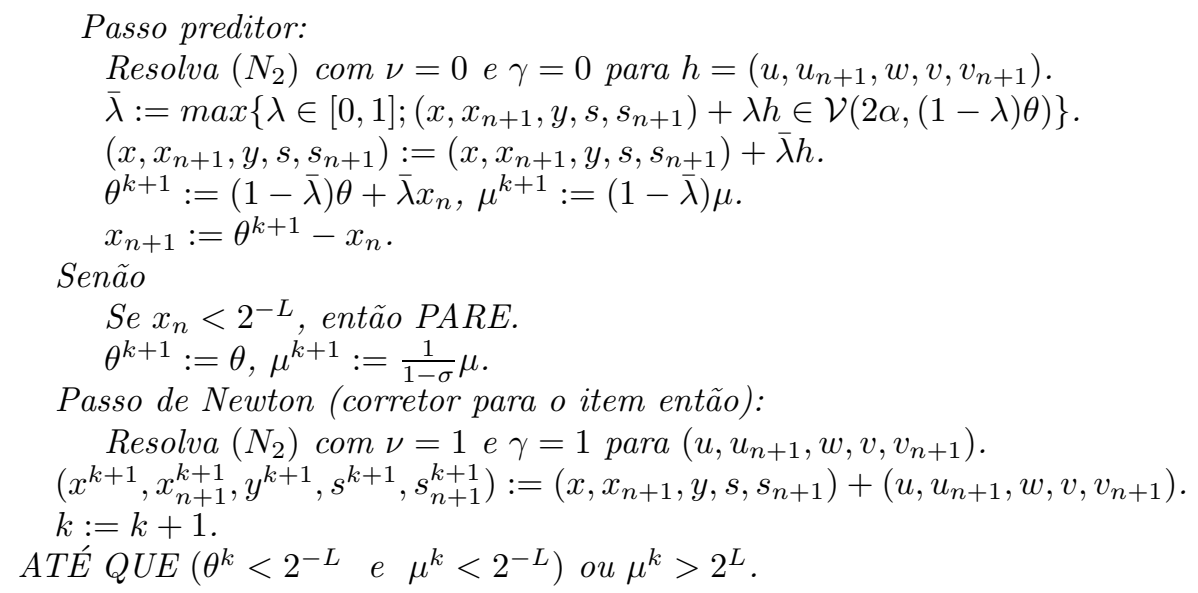

Denotando $v(\tilde{P})$ o valor ótimo de $(\tilde{P})$, a interpretação para a primeira linha no item 'senão' dos Algoritmos 4.1 e 4.2 é a seguinte: resolver o problema $\left(P_{0}\right)$, $\operatorname{logo}(\tilde{P})$, significa encontrar $x$ tal que $c^{T} x \leq v(\tilde{P})+2^{-L}$ e $\theta<2^{-L}$ ou certificar que o problema original é inviável. Assim, se $\theta<2^{-L}$ e estamos no item 'senão' do algoritmo, então o problema original (primal) está resolvido. Ainda, se $\mu>2^{L}$, então o algoritmo certifica que o problema original $(\tilde{P})$ é inviável; apesar da hipótese $(H 1)$.

O próximo resultado é fundamental para a convergência e para a complexidade em iterações do Algoritmo 4.2.

Teorema 4.3. Para toda iteração $k, k \geq 0$, do Algoritmo 4.2,

$$
\delta_{\theta^{k}}\left(x^{k}, x_{n+1}^{k}, s^{k}, s_{n+1}^{k}, \mu^{k}\right) \leq \alpha e\left(x^{k}, x_{n+1}^{k}, s^{k}, s_{n+1}^{k}\right) \in \hat{\mathcal{F}}_{\theta^{k}}^{0} .
$$

Demonstração. Esta demonstração é usual para métodos de pontos interiores (veja, por exemplo, [2]), uma vez que ainda podemos usar o Lema 3.2 de acordo com o passo corretor do item 'então' do algoritmo.

A partir do Teorema 3.3, a convergência do Algoritmo 4.2 é garantida, porque usamos a mesma vizinhança do Algoritmo 4.1 que converge, conforme [3]. Finalmente, o próximo teorema mantém a complexidade em iterações do Algoritmo 4.1, porque a razão do decréscimo referente ao parâmetro $\theta$, isto é, $\theta^{k+1} / \theta^{k}$, no item 'então' do Algoritmo 4.2 é ainda maior do que $(1-\sigma / 4)$. Observe que podemos tomar o máximo entre $\sigma$ e $\bar{\lambda}$ em cada iteração do item 'então'.

Teorema 4.4. O Algoritmo 4.2 pára em, no máximo, $O(\sqrt{n} L)$ iterações.

Abstract In this work we study a dual feasible infeasible-interior-point algorithm for linear programming by Menezes and Gonzaga [3]. We develop a predictorcorrector strategy under certain condition associate to primal feasibility. Our objective is to improve the efficiency of the algorithm, while keeping its complexity in iterations. 


\section{Referências}

[1] L.M.G. Drummond, "Classical and Generalized Central Paths with Algorithmic Applications in Linear Programming", Tese de Doutorado, IMPA/CNPq, 1997.

[2] C.C. Gonzaga, Path-following methods for linear programming, SIAM Review, 34, No. 2 (1992), 167-224.

[3] M.A.F. Menezes, "Um Algoritmo de Ponto-Interior-Inviável com Complexidade $O(\sqrt{n} L)$ Iterações para Programação Linear", Tese de Doutorado, COPPE/UFRJ, 1998.

[4] S. Mizuno, M.J. Todd e Y. Ye, A surface of analytic centers and primaldual infeasible-interior-point algorithms for linear programming, Mathematics of Operations Research, 20, No. 1 (1995), 135-162.

[5] R. Sheng e F.A. Potra, A quadratically convergent infeasible-interior-point algorithm for LCP with polynomial complexity, SIAM Journal on Optimization, 7, No. 2 (1997), 304-317.

[6] Y. Ye, An $O(\sqrt{n} L)$-iteration combined phase I-phase II potential reduction algorithm for linear programming, Department of Management Sciences, The University of Iowa, Iowa City, Iowa 52242, 1992.

[7] Y. Ye, On homogeneous and self-dual algorithms for LCP, Mathematical Programming 76 (1997), 211-221.

[8] Y. Ye, M.J. Todd e S. Mizuno, An $O(\sqrt{n} L)$-iteration homogeneous and selfdual linear programming algorithm, Mathematics of Operations Research, 19, No. 1 (1994), 53-67. 
